a young and rapidly growing subject will inevitably date rather quickly, and it so happened that the launching of the first Russian Earth satellite occurred at a very late stage in the preparation of this work. The preliminary account of the Russian results, and the rapid switch in the emphasis of the American satellite programme from the Vanguard to the Explorer series, are dealt with rather briefly in annexes. It is perhaps fortunate that we have on record here, written before it was overtaken by events, the American plan for the scientific programme intended for the Vanguard satellites.

Viewed as a whole the volume cannot fail to be an invaluable source of reference to workers in the field. The upper atmosphere research rocket which still has a vital part to play is not neglected, but the greater part of the book is devoted to Earth-satellite programmes and plans. Those whose interest is more general will gain an insight into the complexities of planning and the widespread co-operation needed in a space research programme, in addition to a sober review of the many new avenues of scientific research now being opened. The volume takes the form of a series of scientific papers, covering subjects as diverse as the design of instruments for many rocket- and satellite-borne experiments, and the organization of volunteer visual observing teams, both in the United States and in the U.S.S.R. It is indeed pleasing to find an international flavour throughout, with significant contributions from the U.S.S.R.

The book is handsomely produced and illustrated, as is to be expected for the price. One must hope that the end of the International Geophysical Year itself will not prevent the compilation of further authoritative international volumes in these expanding fields.

\section{MICROCOSM TO MACROCOSM}

Matter, Earth and Sky

By Prof. George Gamow. Pp. xi +593 . (London : Macmillan and Co., Ltd., 1959.) 50s. net.

$\mathrm{E}$ XUBERANT and encyclopædic are the only terms to describe this interpretation of the material universe, most appropriately dedicated to "Aspiring Youth". In the days of stone-turning and avenue-exploring, barriers had to be either surmounted or torn down; Prof. Gamow himself was the first to realize that with the right kind of approach nothing so drastic is needed, and that they can be gently tunnelled through. There is certainly a barrier between the experience of the ordinary individual and the physicist's interpretation of it. In this book, without demanding excessive penetration on the part of the reader, the author has successfully brought into the open the truths that occupy the inner levels of the well.

The style follows the author's usual successful formula-saying what comes naturally. There are some old friends, including C. G. H. Tompkins, translated to an American setting, but still on a communal Cambridge bicycle. The illustrations are excellent, original and relevant-oven those that are put in just for fun, like the one showing an 'experi. ment' on the thermal expansion of a body, which looks rather unkind. Scientists are pictured with a richly human touch-Otto Hahn registering sheer amazement at the fission of uranium, Compton strumming his banjo with effect, and Bohr in orbit on a motor-cycle.

The book is divided into three sections. The first, on "Matter and Energy", deals with the elementary physics of the surroundings, relating everyday observations to fundamental principles. This ranges widely from simple mechanics to computers and satellites and rocketry and relativity. The second, entitled "Microcosm", starts with the kinetic view of matter in terms of molecules, and covers atomic and nuclear physics, and a good deal besides. The chapter on the chemistry of life, which goes very fully into protein structure, and discusses Watson and Crick's model of the structure of deoxyribonucleic acid and its implications for the possible working of heredity, is important both for its contents and its influence on the young reader who may (though not if he has got so far through the book) tend to regard physics as a little remote from living. The third part, "Macrocosm", deals with the Earth and its history, the planets, the evolution of the stars, the origin of the elements and of the galaxies, and the recent theory of continuous creation.

So much could not have been achieved in a single book without very careful planning of the sequence of material, and much skill has been devoted to placing the discussion of a fundamental topic in relation to the general framework. Electrolysis, for example, appears in the second part, where the electrical nature of matter is treated. This means that it is a book to be read through, without dodging back to the beginning for explanations.

It is a splendid book, and highly to be recommended to the general reader and for the library. Although very good value for money, its price unfortunately places it high up in the gift-book class. The unkind experiment mentioned above supposes the co-opera. tion of a good-natured relative, and any aspiring youth who can get such a one in an expansive mood would be well advised to clamour for this book as a present instead.

G. R. NOAKES

\section{SURVEYS IN 'APPLIED' MATHEMATICS}

Some Aspects of Analysis and Probability

By Irving Kaplansky, Marshall Hall, Jr., Edwin Hewitt and Robert Fortet. (Surveys in Applied Mathematics, Vol. 4.) Pp. xi +243 . (Now York: John Wiley and Sons, Inc.; London: Chapman and Hall, Ltd., 1958.) 72s. net.

7 HIS book is the fourth of John Wiley's "Surveys in Applied Mathematics"; its production was sponsored by the United States Office of Naval Research and by the editorial board of "Applied Mechanies Reviews"; its authors are described as being, and indeed are, "internationally recognized authorities in the areas of applied mathematics covered by their surveys": Hall writes on combinatorial analysis, and Fortet on probability theory, while functional analysis and abstract harmonic analysis are surveyed respectively by Kaplansky and Hewitt. An English reader, accustomed to the rigid division between applied mathematies (where nothing is proved) and pure mathematics (where nothing is useful) may well find the classification puzzling. Only the probabilist will be untroubled by it; his subject, having an axiomatic foundation and yet deriving 
all its inspiration from practical problems, has no place in the traditional classification; he will be quite happy in the applied mathematical club so long as the topological-algebraists are there to keep him company.

Kaplansky's article (32 pages) is the shortest in the book, but is supported by a magnificent bibliography of 113 entries, nearly half of them concerning papers by Russian authors; he gives a remarkably clear and concise account of many topics of eurrent interest in the theories of Banach spaces, locally convex spaces and Banach algebras.

Hall's survey of combinatorial analysis will be of value not only to algebraists but also to statisticians interested in the existence and construction of designs and to the industrial mathematician concerned with linear programming. For the latter there is a fascinating chapter on the theorems of Philip Hall, König and Ramsey, and their applications; these include the transportation problem, the travelling-salesman problem, and also such curiosities as the following (Erdős and Szekeres, 1935): "There exists an integer-valued function $N(n)$ of the integer variable $n$ such that every set of $N$ points in the plane, no three on a line, will contain $n$ points forming a convex $n$-gon".

Hewitt's long article on abstract harmonic analysis presents a connected account of a difficult and important field, which is the more valuable because a large number of the most important papers reviewed here were published in Russian. Readers of the older text-books on Lebesgue integration encounter theorems of two kinds : those which make essential use of the group structure of the real line, and those which do not. Those which do not (for example, the Lebesgue convergence theorems) properly belong to measure theory, while those which do (for example, theorems about convolutions, and the whole of the Fourier theory) can nearly all be generalized to the situation in which the real line is replaced by a (say, abelian) locally compact group and Lebesgue measure is replaced by Haar measure, and this is the situation with which abstract harmonic analysis is concerned.

Fortet gives a most valuable account of a number of special topies in probability theory, of which the most characteristic is development of techniques for handling random elements of general type. A probability space is a non-vacuous set $\Omega$, a Borel field 7 of 'measurable' subsets of $\Omega$, and a totally finite measure $\mu$ on 7 normalized so that $\mu(\Omega)=1$. A random variable $x($.$) is a mapping from \Omega$ to the real line such that all counter-images of real intervals are 7-measurable, and an n-dimensional random variable is defined similarly. The classical theory was concerned exclusively with finite sets of such random variables; the modern theory of stochastic processes is concerned with infinite sets of random variables, suitably parametrized, and the theory of general random elements is concerned with (in the first instance single) random variables $x($. where now the range of the mapping $\omega \rightarrow x(\omega)$ lies not on the real line nor in $n$-dimensional euclidean space, but in some more general topological-algebraic structure. For example, Mourier and Fortet have studied random variables taking values in a Banach space, and Gel'fand has studied random (Sehwartz) distributions. In a sense the distinction between stochastic processes and general random elements is artificial, for if $\omega \rightarrow x(t, \omega)$ (for each $t$ in some parameter set $T^{\prime}$ ) is a set of random variables constituting a stochastic process, then $\omega \rightarrow x(., \omega)$ can be thought of as a general random element, and conversely most of the general random elements one wishes to consider in practice (random age-distributions, random energy-spectra, etc.) can be reduced to systems of numerical random variables. But the direct treatment of a random variable of general type, where possible, offers many attractions, and there can be no doubt that this branch of the subject will attract considerable attention during the next few years.

The publishers are to be congratulated both on the quality of the surveys included in this volume and on their decision to publish this group of four surveys together.

D. G. KEndali

\section{FUTURE MARINE BIOLOGICAL RESEARCH}

\section{Perspectives in Marine Biology}

A Symposium held at Scripps Institution of Oceanography, University of California, March 24-April 2, 1956. Edited by A. A. Buzzati-Traverso. Pv. xvi+ 621. (Berkeley and Los Angeles: University of California Press; London: Cambridge University Press, 1958.) 75s. net.

"A PPRoximately 90 to 95 per cent of all biologists are engaged in terrestrial biology. ... These biologists have a tendency to consider Marine Biology as a somewhat secondary biological annex." P. Drach (p. 603 et seq.) further states that general biology can never be properly balanced if based predominantly on terrestrial forms, however important they are to our welfare, a view shared by many of the contributors. The expansion of marine biology is reflected by the wide range of subjects presented at a symposium planned to focus attention on fortheoming fields of marine research. It is significant that a number of contributions come from non-marine biologists. Forty-one papers, with subsequent discussions, are arranged in four sections : ecology; physiology and biochemistry; behaviour; evolution and genetics.

Ecological papers by P. Drach, L. Zenkovitch, G. Thorsen, A. C. Hardy and others, while emphasizing that more field observations on animal communities are required, show the urgent need for new design and international standardization of instruments and techniques for quantitative sampling. Field observation is so much stressed that K. M. Rae's plea, echoed elsewhere, for laboratory observation and experiment on marine equivalents to the guinea pig and fruit fly stands out from the rest. D. P. Wilson's studies on the ability of organisms to detect factors at present defying analysis, and so select a particular substrate, are stimulating. Further emphasis on this need for study on microconstituents is given by S. K. Kon (vitamins and external metabolites), D. I. Arnon (micronutrients) and L. Provasoli (growth factors of marine algae). E. Baldwin's paper on biochemical perspectives and the need for more biochemists in marine research is timely.

Papers on reef-building corals (C. M. Yonge); productivity, patchiness and succession in plankton (W. Rodhe et al., R. Margalef, A. C. Hardy, L. Tonelli and V. Tonelli); cell chemistry (E. S. G. Barron, A. Szent-Györgyi); biological clocks (C. S. Pitten- 